\title{
Towards Interactive Planning of Coil Embolization in Brain Aneurysms
}

\author{
Jeremie Dequidt ${ }^{1}$, Christian Duriez ${ }^{1}$, Stephane $_{\text {Cotin }}{ }^{1}$, and Erwan Kerrien ${ }^{2}$ \\ 1 INRIA Lille Nord Europe - Alcove Team \\ 50, avenue Halley 59655 Villeneuve d'Ascq, France \\ \{jeremie.dequidt, christian.duriez, stephane.cotin\}@lifl.fr \\ 2 INRIA Nancy Grand Est - Magrit Team \\ Campus Scientifique, 54506 Vandoeuvre-lès-Nancy, France \\ erwan.kerrien@loria.fr
}

\begin{abstract}
Many vascular pathologies can now be treated in a minimally invasive way thanks to interventional radiology. Instead of open surgery, it allows to reach the lesion of the arteries with therapeutic devices through a catheter. As a particular case, intracranial aneurysms are treated by filling the localized widening of the artery with a set of coils to prevent a rupture due to the weakened arterial wall. Considering the location of the lesion, close to the brain, and its very small size, the procedure requires a combination of careful planning and excellent technical skills. An interactive and reliable simulation, adapted to the patient anatomy, would be an interesting tool for helping the interventional neuroradiologist plan and rehearse a coil embolization procedure. This paper describes an original method to perform interactive simulations of coil embolization and proposes a clinical metric to quantitatively measure how the first coil fills the aneurysm. The simulation relies on an accurate reconstruction of the aneurysm anatomy and a real-time model of the coil for which sliding and friction contacts are taken into account. Simulation results are compared to real embolization procedure and exhibit good adequacy.
\end{abstract}

\section{Introduction}

Intracranial aneurysms are abnormal bulges of the arterial wall that may occasionally rupture, causing hemorrhage, stroke or death. The coil embolization technique involves approaching the aneurysm by navigating through the vascular network using a catheter until the diseased blood vessel is reached, thus avoiding the need to open the skull. Once the aneurysm is reached, a micro-catheter is then partially inserted inside the aneurysm, and the coil is carefully deployed using a guidewire pushing the coil out of the micro-catheter. Usually, several coils are deployed to fill the aneurysm, allowing to clot and eventually to stop blood from entering the aneurysm.

Although the overall morbidity and mortality associated with endovascular repair is lower than for surgery (9.5\% at one year, compared to $12.2 \%)$ the 
technical challenges associated with coil embolization remain important. It was however shown recently that the risk of complications with coil embolization of unruptured aneurysms decreases dramatically with physician experience [1]. Indeed, complete embolization is usually determined by subjective assessments of radiologists. Although several objective criteria have recently been introduced to help radiologists during the procedure 2 simulation systems can offer more functionalities and can be used for physician training as well as for pre-operative planning.

Previous work in the area of real-time or near real-time simulation for interventional radiology has mainly focused on training. For instance, Li et al. [3], Hoefer et al. 4, Alderliesten et al. 5, or Cotin et al. 6] 7] have proposed different approaches for modeling either catheter deformation and more generally catheter navigation in vascular networks. Regarding the planning of interventional radiology procedures, the most representative work was in the area of stent deployment. Laroche et al. 8 , proposed a finite element model for a patientspecific planning of balloon angioplasty and stent implantation. Complex deviceartery interactions occurring during stent deployment were computed, however the simulation was not performed in real-time.

The objective of the work described in this paper is to provide a tool that can be used for planning the procedure. This involves selecting the proper characteristics of the coil based on the patient anatomy, but also interactively deploying the virtual coil(s) to plan or rehearse the procedure. The interactive aspect in this planning plays a key role as the interventional radiologist constantly controls the motion of the coil and as such influences the final coil placement within the aneurysm.

Section (2) presents the different models used for the simulation: the reconstruction of vascular networks based on rotational angiography and the coil model based on beam elements. The contact modeling to tackle interactions between the aneurysm and the coils as well as self-collision of the coils are described in section (3) which also introduces the optimizations allowing interactive simulations of coil embolization procedure. Finally, simulation results are presented in section (4) where we introduce a clinical metric in order to compare the simulation to actual coil deployments in patient-specific aneurysms.

\section{Models}

A complete coil embolization consists in coils with specific rest shapes conforming to the geometry of an aneurysm. To reproduce this matching problem within a simulation, accurate models are required. This section describes the modelling stage of our framework which aims at virtually reproducing the most important features of real data (such as geometry for the vascular network or mechanical properties for the coils).

Patient-Specific Aneurysm Model. The input of our algorithm is a volumetric $3 \mathrm{D}$ Xray (3DXA) angiography of the brain. Such $3 \mathrm{D}$ images are recognized 
as being of a daily clinical usefulness for the planning and follow-up of the treatment of cerebral pathologies 9. In order to use deformable active surfaces to segment the aneurysm, an initial surface has to be defined. This region of interest is obtained by thresholding the volumetric data with an appropriate value given by the user. The triangulated initial surface is the boundary of this region and is classically obtained using a marching cube algorithm 10. This first guess is obviously not accurate due to the use of a global threshold. Deformable triangulated surfaces are then used to recover a precise shape of the aneurysm starting from the initial shape.

The method used for the $3 \mathrm{D}$ reconstruction of the aneurysm shape from the initial guess $V$ originates in [1]. Let $v_{i}$ be the vertices of the triangulated mesh. The shape of the aneurysm is defined as the set of vertices $V$ which minimizes the energy term $E=\sum_{i=1}^{n} E_{\text {internal }}\left(v_{i}\right)+\lambda E_{\text {external }}\left(v_{i}\right)$ where $E_{\text {internal }}\left(v_{i}\right)=$ $\left|v_{i}-\bar{v}_{i}\right|$ imposes smoothness constraints on the surface $\left(\bar{v}_{i}\right.$ being the average set of vertices connected to $v_{i}$ ) and $E_{\text {external }}$ is the force field created by the image gradient: $E_{\text {external }}\left(v_{i}\right)=-\left|\nabla I\left(v_{i}\right)\right|$ where $I\left(v_{i}\right)$ is the 3DXA intensity at vertex $v_{i}$.

Using active surfaces not only improves the segmentation of the aneurysm but also provides a smooth surface representation, presenting better characteristics for further processing during the simulation. In particular, the computation of the interaction between the coil and aneurysm wall requires a smooth surface. We obtain a more regular representation than what can be obtained using a marching cubes algorithm [10, even when applying a post-processing smoothing.

Coil Model. There are different types of detachable coils but most of them have a core made of platinum, and some are coated with another material or a biologically active agent. All types are made of soft platinum wire of less than a millimeter diameter and therefore are very soft. The softness of the platinum allows the coil to conform to the arbitrary shape of an aneurysm.

The deformation model of the coil is based on the recent work of Dequidt et. al. 12. Coil dynamics is modelled using serially linked beam elements:

$$
\mathbf{M} \dot{\mathbf{v}}=\mathbf{p}-\mathbb{F}\left(\mathbf{q}, \mathbf{v}, \mathbf{q}_{0}\right)+\mathbf{H} \mathbf{f}
$$

where $\mathbf{M} \in \mathbb{R}^{(n \times n)}$ gathers the mass and inertia matrices of the beam elements. $\mathbf{q} \in \mathbb{R}^{n}$ is the vector of generalized coordinates (each node at the extremity of a beam has six degrees of freedom: three of which correspond to the spatial position, and three to the angular position in a global reference frame). The rest position $\mathbf{q}_{0}$ depends on the family of coil to be simulated: helical shape or 3D shape consituted of omega loops (see figure 1). $\mathbf{v} \in \mathbb{R}^{n}$ is the vector of velocity. $\mathbb{F}$ represents internal visco-elastic forces of the beams, and $\mathbf{p}$ gathers external forces. $\mathbf{f}$ is the vector of the contact forces with the aneurism wall, and $\mathbf{H}$ gathers the contact directions. To integrate this model we use backward Euler scheme with a unique linearization of $\mathbb{F}$ per time step. 


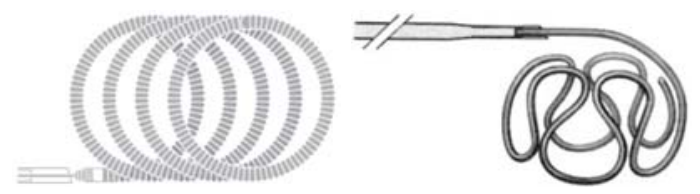

Fig. 1. Example of coils used in our simulations, left: Boston Scientific helical coil GDC 10, right: 3D GDC built with omega loops [13.

\section{Simulation of Coil Deployment}

\subsection{Modeling Contacts with Aneurysm Walls}

Simulating coil embolization requires to accurately model contacts that occur between the coil and the wall of the aneurysm. This contact model must account for the stick and slip transitions that take place during the deployment of the coil. The model also includes a compliant behavior of vessel wall that is close to Boussinesq model [14]. For modeling contacts with friction, we use two different laws, that are based on the contact force and on the relative motion between the coil and the aneurysm wall. The contact law is defined along the normal $\mathbf{n}$ and the friction law, along the tangential $(\mathbf{t}, \mathbf{s})$ space of the contact.

The contact model, based on Signorini's law, indicates that there is complementarity between the gaps $\delta^{n}$ and the contact forces $\mathrm{f}^{n}$ along the normal direction, that is:

$$
0 \leq \delta^{n} \perp \mathrm{f}^{n} \geq 0
$$

With Coulomb's friction law, the contact force lies within a spacial conical region whose height and direction are given by the normal force, giving two complementarity conditions for stick and slip motion:

$$
\begin{array}{ll}
{\left[\begin{array}{ll}
\delta^{t} & \delta^{s}
\end{array}\right]=\mathbf{0} \Rightarrow\left\|\left[\begin{array}{ll}
\mathrm{f}^{t} & \mathrm{f}^{s}
\end{array}\right]\right\|<\mu\left\|\mathrm{f}^{n}\right\| \quad} & \text { (stick condition) } \\
\left.\left[\begin{array}{ll}
\delta^{t} & \delta^{s}
\end{array}\right] \neq \mathbf{0} \Rightarrow\left[\begin{array}{ll}
\mathrm{f}^{t} & \mathrm{f}^{s}
\end{array}\right]=-\mu\left\|\mathrm{f}^{n}\right\| \frac{\left[\begin{array}{ll}
\delta^{t} & \delta^{s}
\end{array}\right]}{\|\left[\delta^{t}\right.} \delta^{s}\right] \| & \text { (slip condition) }
\end{array}
$$

Where the vector $\left[\begin{array}{ll}\delta^{t} & \delta^{s}\end{array}\right]$ provides the relative motion in the tangential space and $\mu$ represents the friction coefficient.

The obtained complementarity relations could create singular events when it changes from one state to an other: For instance, when a collision occurs at instant $t^{\star}$, the velocity $\mathbf{v}\left(t^{\star}\right)$ of the coil, at that point, changes instantaneously. The acceleration could then be ill-defined and we can observe some quick changes in the dynamics. Each friction contact creates three nonholonomic constraints along the normal and tangential directions. Our approach allows for processing simultaneously multiple friction contacts, including self-contacts on the coil.

\subsection{Simulation Steps}

The processing of one simulation step begins by solving equation (1) for all forces except contact forces $(\mathbf{f}=0)$. This free motion corresponds essentially to 
the deformation of the beam elements under gravity and user force input. Once the free motion has been computed, collision detection computes the contact points between the coil model and the aneurysm surface and the points of selfcollision. When collisions are detected, the contact response is computed. This is a complex aspect that influences greatly the overall behavior of the coil model. To describe the mechanical behavior during contact, the mechanical coupling between the different contact points is modeled. This information is provided by evaluating the compliance matrix in the contact space, called $\mathbf{W}$, for both the coil and the aneurysm. Let's consider a contact $\alpha$ on the node $i$ of the coil (with one constraint along the contact normal $\mathbf{n}$ and two along the tangential friction directions $\mathbf{t}, \mathbf{s}) . \mathbf{H}_{\alpha}$ is the matrix of the frame $[\mathbf{n} \mathbf{t} \mathbf{s}]$. The mechanical coupling of this contact with a contact $\beta$ (with frame $\mathbf{H}_{\beta}$ ) on node $j$ can be evaluated with the following $3 \times 3$ matrix:

$$
\mathbf{W}_{(\alpha, \beta)}=\mathbf{H}_{\alpha}^{T}\left(\frac{\mathbf{M}}{h^{2}}+\frac{d \mathbb{F}}{h d \mathbf{v}}+\frac{d \mathbb{F}}{d \mathbf{q}}\right)_{(i, j)}^{-1} \mathbf{H}_{\beta}=\mathbf{H}_{\alpha}^{T} \mathbf{C}_{(i, j)} \mathbf{H}_{\beta}
$$

where $\mathbf{C}_{(i, j)}$ is the $3 \times 3$ sub-matrix of global compliance matrix $\mathbf{C}$ (inverse of tangent matrix) at the rows of node $i$ and the columns of node $j$. For the aneurysm wall, the formulation of the coupling is simpler:

$$
\mathbf{W}_{(\alpha, \beta)}=\frac{g\left(d_{i j}\right)}{e} \mathbf{H}_{\alpha}^{T} \mathbf{H}_{\beta}
$$

where $e$ is an elasticity parameter that is homogeneous to Young modulus and $g\left(d_{i j}\right)$ is a Gaussian function of the distance, defined on the surface, between contact points $i$ and $j$. The Gaussian function allows a fall-off of the coupling with increasing distance between the contact points. This model is close to Boussinesq's approximation which provides a distribution of the normal contact stress from the elasticity of the surface, around a point of contact [14].

The result of the contact response consists in finding the friction contact forces that respect Signorini's and Coulomb's laws. Several works [15] or 6] present Gauss-Seidel iterative approaches that solve this problem. The solver needs an evaluation of a global compliance matrix $\mathbf{W}$, which is the sum of the compliances of the coil and the aneurysm wall. It also needs the value of the relative displacement of the contacting points during the free motion $\boldsymbol{\delta}^{\text {free }}$. When the contact forces are found, during the last step, called contact correction we compute the motion associated to the contact forces.

\section{Validation and Results}

The main contribution of this work is not to provide an extensive study on simulated coil deployments but rather to introduce a global approach, from segmentation to simulation of the coil embolization procedure. This approach includes a validation step, for which we propose an adapted clinical metric. Examples provided in the paper serve as preliminary results and exhibit the relevance of the chosen metric. 


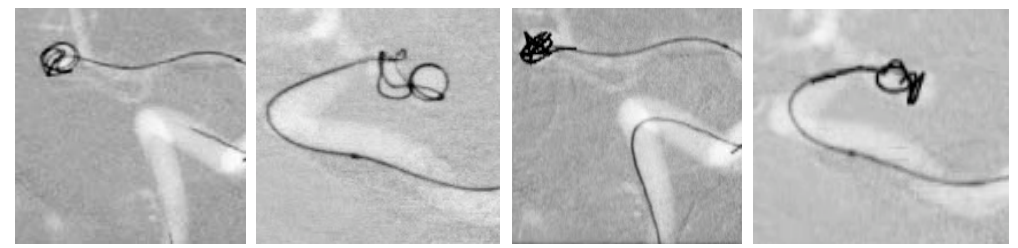

Fig. 2. Examples of our simulation results: (left) real coil embolization (right) our simulated coil embolization with $3 \mathrm{D}$ coils

Clinical Metric. As pointed out in the introduction, a successful embolization procedure is determined by the subjective assessment of radiologists and an objective measure known as Coil Embolization Ratio (CER) 2]. CER quantifies the amount of coil material that fills up the aneurysm volume. Usually a CER between $20 \%$ and $33 \%$ is a good value for a complete embolization. The validation proposed in this paper focuses on the deposit of the first coil, which is known to be the most difficult to place and plays a key role in the procedure as it serves as a framework for further embolization. Comparison between final shapes of the real coil and simulated coils is not a suitable solution in this context. Indeed coil deployments are hardly reproducible even with the same initial conditions because they depend of many parameters such as radiologist input, irregularities of the aneurysm surface and can lead to very different final shapes (thus justifying the clinical use of CER for complete embolization). Even experienced interventional radiologists can hardly estimate how the first coil will finally be placed inside the aneurysm. They do however have some criteria to select the first coil [16]: an appropriate size (diameter of the coil has to be close to the aneurysm fundus and larger than the neck), its deployment should cover a large surface of the aneurysm (to serve as a basket in which the subsequent coils will be deposited) and some loops should extend across the neck of the aneurysm. Therefore, we propose in this paper to use a clinical metric derived from the work of Cloft et. al. [16]: the coil embolization surface ratio (CESR) that defines the amount of the aneurysm wall covered by the first coil. The choice of the CESR is twofold: first, it translates into a metric the qualitative criteria proposed by Cloft et. al. and second is less sensitive to small perturbations that could lead to very different shapes. During the beginning of the embolization procedure, the physician constantly evaluates the CESR by watching the 2D fluoroscopic image of the coil deployment. We can obtain easily a measure of CESR and CER of the simulation results but the lack of $3 \mathrm{D}$ view of the real coil deployment prevents from having a precise metric on patient data. For a preliminary assessment, we perform the validation of the simulation data by evaluating the CESR on 2D fluoroscopic views as it is widely done by neuroradiologist during procedure.

Simulations. The simulations have been performed using two reconstructed patient aneurysms. For each aneurysm, deployments of a first coil have been simulated. The coil was modeled using 200 beam elements with Young modulus $=75 \mathrm{GPa}$ and Poisson ratio $=0.39$. The smooth surface model of the aneurysm 
Table 1. CESR measure for the two procedures. Comparable CESR values are obtained when using the same coil as the real procedure. When simulating a helical coil (which is not relevant for this shape of aneurysm), we notice a lower CESR, which is consistent with what would happen in an actual procedure. This shows the predictive nature of our simulation.

\begin{tabular}{|c|c|c|}
\hline & CSER & Error in \% \\
\hline First Patient, experimental data (3D Coil) & $81 \%$ & 0 \\
First Patient Aneurysm with 3D Coil & $68 \%$ & 16 \\
First Patient Aneurysm with Helical Coil & $55 \%$ & 32 \\
\hline Second Patient, experimental data (3D Coil) & $24 \%$ & 0 \\
Second Patient Aneurysm with 3D Coil & $17 \%$ & 30 \\
Second Patient Aneurysm with Helical Coil & $11 \%$ & 54 \\
\hline
\end{tabular}

walls allowed for the simulation of the fast stick and slip transitions that can be observed in actual coil embolizations. Using the shape functions of the beam elements to interpolate the contact points, we obtain a precise auto-collision response. The implicit integration of the coil dynamics allows for a stable behavior of the coil model even during highly constrained transitory motions. The computation time is quite fast at the beginning of the simulation $(30 \mathrm{~ms}$ for the first loops) but slows down when the contact points become numerous. In order to provide the same initial conditions for the real procedure and the simulated one, the catheter tip is positioned and oriented using a $3 \mathrm{D}$ triangulation of $2 \mathrm{D}$ orthogonal fluoroscopic images allowing good accuracy $(0.2 \mathrm{~mm})$ and is less invasive on the deployment than EM trackers. The simulation of the coil is interactive and is controlled using an optical device to acquire the motion induced made by the user. The blood flow resistance has not been taken into account as its influence is said to be negligible by radiologists.

Error Measurement. Our validation tests consist in measuring the CSER metric and computing the relative error with respect to the value obtained from experimental data. The CSER of the experimental data is estimated from $2 \mathrm{D}$ images so is a bit overvalued compared to the CSER of our simulations where an accurate area computation of the aneurysm volume covered by coils can be obtained. A summary of the results appears on table 1 and exhibits good adequacy of the behavior of our simulated coils with respect to real ones: indeed our simulated 3D coils provide CSER values close to the experimental values whereas helical coils induce a lower CESR which is consistent with what would happen in an actual procedure.

\section{Conclusion and Future Work}

In this paper, we have presented a global approach for performing simulation of coil embolization on a patient dataset. The geometric reconstruction of the aneurysm shape is adapted to the simulation requirements. An efficient dynamic coil model is combined with physically based processing of contacts with the 
aneurysm wall and the coil itself. The methodology includes a validation process, based on clinical metric. The preliminary results we have obtained are very encouraging, as they illustrate the potential of our approach to capture the key characteristics of this complex procedure and demonstrate a behavior that matches experimental data.

Our next step is to optimize the collision detection and response pipeline in order to obtain real-time performance during a simulation of a whole embolization. We also plan to extend the validation on more patients in order to evaluate the potential use of such a simulation during the planning process of a coil embolization.

Acknowledgments. We would like to thank René Anxionnat, Juan Pablo De la Plata and Frederick Roy for their help on this work.

\section{References}

1. Singha, V., Gressa, D., Higashidab, R., Dowdb, C., Halbachb, V., Johnston, S.: The learning curve for coil embolization of unruptured intracranial aneurysms. American Journal of Neuroradiology 23, 768-771 (2002)

2. Satoh, K., Ito, Y., Abe, H.: Measurement of volume ratio to predict coil compaction, on aneurysmal embolization. Interventional Radiology 1(4), 179-182 (1998)

3. Li, Z., Chui, C.-K., Cai, Y., Anderson, J.H., Nowinski, W.L.: Interactive catheter shape modeling in interventional radiology simulation. In: Niessen, W.J., Viergever, M.A. (eds.) MICCAI 2001. LNCS, vol. 2208, p. 457. Springer, Heidelberg (2001)

4. Hoefer, U., Langen, T., Nziki, J., Zeitler, F., Hesser, J., Mueller, U., Voelker, W., Maenner, R.: Cathi - catheter instruction system. In: Computer Assisted Radiology and Surgery (CARS), Paris, France, pp. 101-106 (2002)

5. Alderliesten, T.: Simulation of Minimally-Invasive Vascular Interventions for Training Purposes. PhD dissertation, Utrecht University (2004)

6. Cotin, S., Duriez, C., Lenoir, J., Neumann, P., Dawson, S.: New approaches to catheter navigation for interventional radiology simulation. In: Duncan, J.S., Gerig, G. (eds.) MICCAI 2005. LNCS, vol. 3750, pp. 534-542. Springer, Heidelberg (2005)

7. Dequidt, J., Lenoir, J., Cotin, S.: Interactive contacts resolution using smooth surface representation. In: Ayache, N., Ourselin, S., Maeder, A. (eds.) MICCAI 2007, Part II. LNCS, vol. 4792, pp. 850-857. Springer, Heidelberg (2007)

8. Laroche, D., Delorme, S., Anderson, T., Diraddo, R.: In-vivo validation of a stent implantation numerical model. In: Proc. Medicine Meets Virtual Reality Conference, Studies in Health Technology and Informatics., vol. 125, pp. 265-270 (2007)

9. Anxionnat, R., Bracard, S., Ducrocq, X., Trousset, Y., Launay, L., Kerrien, E.: Intracranial aneurysms: Clinical value of $3 \mathrm{~d}$ digital subtraction Angiography in the Therapeutic Decision and Endovascular Treatment. Radiology (2001)

10. Lorensen, W., Cline, H.: Marching cubes: A high resolution 3d surface construction algorithm. In: Computer Graphics Proceedings, SIGGRAPH, vol. 21, pp. 163-169 (1987)

11. Lachaud, J.O., Montanvert, A.: Deformable meshes with automated topology changes for coarse-to-fine 3D surface extraction. Medical Image Analysis 3(2), 187207 (1999) 
12. Dequidt, J., Marchal, M., Duriez, C., Kerrien, E., Cotin, S.: Interactive simulation of embolization coils: Modeling and experimental validation. In: Proceedings of MICCAI (2008)

13. Cloft, H.J., Joseph, G.J., Tong, F.C., Goldstein, J.H., Dion, J.E.: Use of threedimensional guglielmi detachable coils in the treatment of wide-necked cerebral aneurysms. American Journal of Neuroradiology (1999)

14. Pauly, M., Pai, D., Leonidas, G.: Quasi-rigid objects in contact. In: Proceedings of ACM SIGGRAPH Symposium on Computer Animation, pp. 109-119 (2004)

15. Jourdan, F., Alart, P., Jean, M.: A gauss-seidel like algorithm to solve frictional contact problems. Comp. Meth. In: Appl. Mech. and Engin, 33-47 (1998)

16. Cloft, H.J., Joseph, G.J., Tong, F.C., Goldstein, J.H., Dion, J.E.: Use of threedimensional guglielmi detachable coils in the treatment of wide-necked cerebral aneurysms. American Journal of NeuroRadiology, 1312-1314 (2000) 